\title{
Review of the gyrotron theory
}

\author{
Gregory S. Nusinovich \\ Institute for Research in Electronics and Applied Physics, University of Maryland, College Park, MD 20742-3511, USA, gre- \\ goryn@umd.edu
}

This review is focused on presenting results of the studies of beam-wave interaction in high-power gyrotrons performed during last 15 years by the presenter and his co-authors. All results are gathered in three groups that can tentatively be entitled: "Megawatts and above", "Terahertz and beyond" and "Miscellaneous". All studies were performed using either non-stationary, selfconsistent code MAGY [1], or self-consistent, nonstationary, multimode code based on equations formulated in Ref. [2], or using quasi-linear approach, in which the dependence of the electron current density on the intensity of the RF field is described by polynomials (see, e.g., [3]).

Megawatts and above. In this section the attention is paid, first, to the issue of mode interaction in MW-class gyrotrons operating in very-high-order modes. Results of MAGY simulations [4] illustrated by Fig. 1 demonstrate that the most dangerous are the modes with a certain frequency separation from the operating one. Since the most efficient is the operation in the regime of hard selfexcitation, the most dangerous are the parasitic modes with a lower frequency located in the region of soft selfexcitation, where start currents are small.

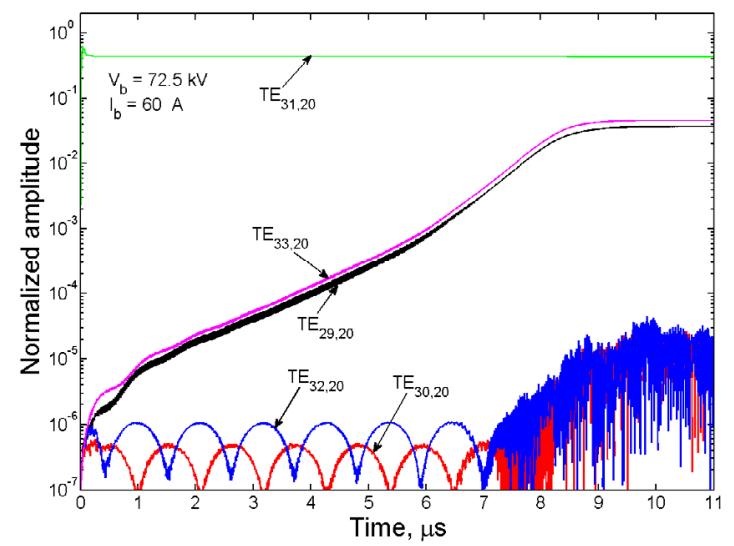

Fig. 1. Excitation of a pair of distant sidebands followed by excitation of a pair of close sidebands in a $170 \mathrm{GHz}$ gyrotron operating in the $\mathrm{TE}_{31,20}$-mode

The second issue requiring a careful consideration is the start-up scenario, i.e. analysis of methods of initial excitation of the desired mode followed by driving this mode into the region of the most efficient operation. As a rule, it requires passing through the region of soft selfexcitation into the region of hard self-excitation. Such scenario in long-pulse and $\mathrm{CW}$ regimes can be realized by varying the external magnetic field, the cathode and mod-anode voltages and the beam current. (Original concept was formulated in Ref. [5]; the review of later studies was given in Ref. [6].) An example of passing through these regions is shown in Fig. 2.
Fast voltage rise. Typically, the cavity fill time is much shorter than the voltage rise time. Therefore it is always assumed that the amplitude of RF oscillations follows a slow voltage rise [5,6]. As shown in Fig. 2, in this case the voltage rise in gyrotrons with diode-type guns will cause, first, oscillations of the parasitic mode with a lower frequency that can be dangerous for diagnostic channels used in plasma installations where the gyrotrons deliver the power. As shown in [7], the excitation of such parasitic modes can be avoided when the voltage rise is fast enough (Fig. 3).

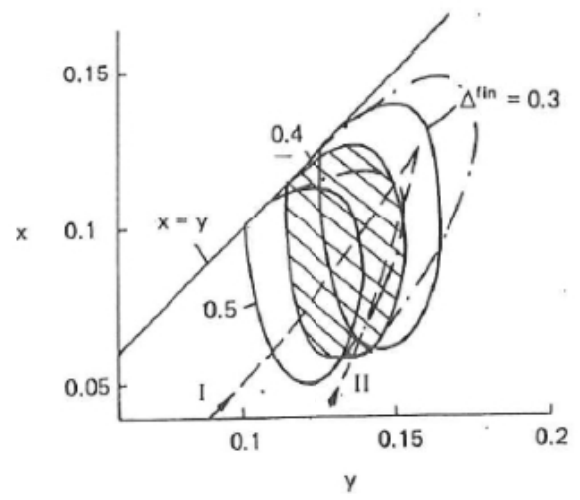

Fig. 2. Horizontal axis shows the total electron velocity squared proportional to the beam voltage; the vertical axis shows the orbital electron velocity squared (this velocity is proportional to the mod-anode voltage). The zone of self-excitation of the operating mode (dashed) is surrounded by similar zones of the highfrequency (lower voltages) and low-frequency (higher voltages) parasitic modes. Gyrotron trace I during the voltage rise corresponds to the diode-type electron gun; the trace II shows the case when during the voltage rise the difference between the beam and mod-anode voltages remains constant
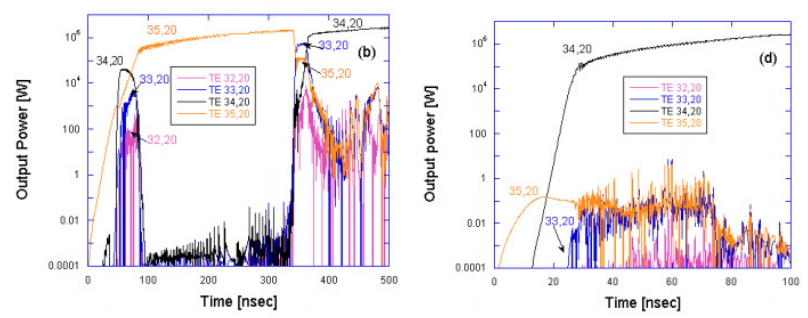

Fig. 3. Excitation of a $170 \mathrm{GHz}$ FZK coaxial gyrotron. The voltage rise from $50 \mathrm{kV}$ to $78 \mathrm{kV}$ is equal to $500 \mathrm{~ns}$ in the left figure and $100 \mathrm{~ns}$ for the right figure

Slow processes. There are known, at least, three processes with typical times much greater than milliseconds: ion neutralization of DC space charge fields of electron beams, thermal expansion of the cavity due to the ohmic losses and, sometimes, beam current reduction due to emission cooling of the cathode. Some possibilities of mitigating these effects were analyzed in Refs. [8, 9] (see also references therein). 
Terahertz and beyond. In this section we discuss, first, the role of such factors in electron beams that become more pronounced with the wavelength shortening, viz., the azimuthal nonuniformity of electron emission and the finite thickness of electron beams. The section also includes consideration of some possibilities of operating at high cyclotron harmonics allowing to increase the wave frequency at given magnetic fields.

Azimuthal nonuniformity. The azimuthal nonuniformity of electron emission reduces the efficiency of gyrotrons and also affects the mode interaction. As known (see, e.g. [3] and references therein) the interaction between two modes is purely amplitude when their frequency separation exceeds the width of their resonance curves $\left(\left|\omega_{2}-\omega_{1}\right|>\omega / Q\right)$ and their azimuthal indices are different $\left(m_{2} \neq m_{1}\right)$; similar conditions can also be written for three-wave interaction.

In the case of azimuthally nonuniform emission the electron current density can be represented as superposition of azimuthal harmonics; thus, certain harmonics can play a role in changing the nature of interaction between the modes. These issues were analyzed in Refs. [10, 11].

Beam thickness. The classical theory of gyrotrons is based on the assumption that an electron beam has no spread in radii of electron guiding centers (a thin annular electron beam). The finite spread causes efficiency degradation and may also affect the mode interaction in soft and hard excitation regimes [12]. For a $670 \mathrm{GHz}$ gyrotron developed jointly by IREAP and IAP these effects were studied in Ref. [13].

A certain contradiction between requirements to the electron spread in guiding center radii and in velocities should be mentioned. For reducing the velocity spread it is beneficial to form quasi-laminar electron beams. However, the radial spread in such beams is relatively large. The trade-off between these requirements, which allows one to optimize the device efficiency, was studied in Ref. [14].

Operation at cyclotron harmonics. Gyrotron operation at cyclotron harmonics has a long history. In recent years, some issues important for operation at cyclotron harmonics were studied in [15-18].

Miscellaneous. This part of the presentation will be devoted to consideration of such effects as after-cavity interaction, efficiency and start currents in gyrotrons with tapered resonators, formation of the axial field structure in open resonators, effect of reflections, mode switching [19] and dynamics and stochasticity in gyrotrons with overlapping of cyclotron resonances [20].

\section{References}

1. M. Botton, T. M. Antonsen, Jr., B. Levush et al.. MAGY: A time-dependent code for simulation of slow and fast wave microwave sources// IEEE Trans. Plasma Sci., 1998,V. 26, No. 3, 882-892.
2. Zavolsky N. A., Nusinovich G. S., Pavelyev A. B., Stability of single-mode oscillations and nonstationary processes in gyrotrons with oversized low-Q resonators // Gyrotrons, edited by V.A. Flyagin, G.S. Nusinovich, A.L. Goldenberg, V.K. Yulpatov, and N. A. Gorodetskaya, IAP AS USSR, 1989, P. 84-112.

3. G.S. Nusinovich, Mode interaction in gyrotrons//Int. J. Electronics, 1981, V. 51, No. 4, 457-474.

4. O.V. Sinitsyn, G.S. Nusinovich and T.M. Antonsen, Jr., Stability of Gyrotron Operation in Very High-Order Modes// Phys. Plasmas, 2012, V. 19, 063114.

5. G.S. Nusinovich, Methods of voltage feeds for a pulsed gyromonotron which ensure high-efficiency in a single-mode operation /Elektron. Teknh., Elektron. SVCh, 1974, No. 3, 44-49.

6. G.S. Nusinovich, O.V. Sinitsyn, L. Velikovich et al., Startup Scenarios in High-Power Gyrotrons// IEEE Trans. Plasma Sci., 2004, V. 32, No. 3, 841-852.

7. G.S. Nusinovich, A.N. Vlasov, T.M. Antonsen, Jr., et al., Excitation of parasitic modes in gyrotrons with fast voltage rise//Phys. Plasmas, 2008, V.15, 103101.

8. G.S. Nusinovich, O.V. Sinitsyn, T.M. Antonsen, Jr. et al., Slow processes in startup scenarios of long-pulse gyrotrons// Phys. Plasmas, 2006, V. 13, 083106.

9. A. Fokin, M.Yu. Glyavin, and G.S. Nusinovich, Effect of ion compensation of the beam space charge on gyrotron operation// Phys. Plasmas, 2015, V. 22, 043119.

10. G.S. Nusinovich and M. Botton, Quasilinear theory of mode interaction in gyrotrons with azimuthally inhomogeneous electron emission// Phys. Plasmas, 2001, V. 8, No. 3, 1029-1036.

11. G.S. Nusinovich, A.N. Vlasov, M. Botton et al., Effect of the azimuthal inhomogeneity of electron emission on gyrotron operation// Ibid., No. 7, pp. 3473-3479.

12. G.S. Nusinovich, O.V. Sinitsyn, M. Yeddulla et al., Effect of the radial thickness of electron beams on mode coupling and stability in gyrotrons// Phys. Plasmas, 2003, V. 10, No. 8, 3335-3343.

13. R. Pu, G.S. Nusinovich, O.V. Sinitsyn, and T.M. Antonsen, Jr. et al., Effect of the thickness of electron beams on the gyrotron efficiency// Phys. Plasmas, 2010, V. 17, 083105; also, R. Pu, G.S. Nusinovich, O.V. Sinitsyn, and T.M. Antonsen, Jr., Numerical study of efficiency for a $670 \mathrm{GHz}$ gyrotron// Phys. Plasmas, 2011, V. 18, 023107.

14. O. Dumbrajs and G.S. Nusinovich, On optimization of sub-THz gyrotron parameters// Phys. Plasmas, 2012, V. 19, 103112 .

15. G.S. Nusinovich, D.G. Kashyn, and T.M. Antonsen, Jr., Harmonic gyrotrons operating in high-order symmetric modes// Appl. Phys. Lett., 2015, V. 106, 013502.

16. G.S. Nusinovich, R. Pu, and V.L. Granatstein, Suppression and nonlinear excitation of parasitic modes in harmonic gyrotrons// Appl. Phys. Lett., 2015, V. 107, 013501.

17. M.Yu. Glyavin, A.G. Luchinin, V.N. Manuilov and G.S. Nusinovich, Design os a dub-THz, Third-Harmonic, Continuous-Wave Gyrotron// IEEE Trans. Plasma Sci., 2008, V. 36, NO. 3, 591-596.

18. M.Yu. Glyavin, N.A. Zavolsky, A.S. Sedov, and G.S. Nusinovich, Low-Voltage Gyrotrons// Phys. Plasmas, 2013, V. 20, 033103.

19. G.S. Nusinovich,O.V. Sinitsyn, and T.M. Antonsen, Jr., Mode switching in a gyrotron with azimuthally corrugated wall// Phys. Rev. Lett., 2007, V. 98, 205101.

20. G.S. Nusinovich, R. Ngogang, T.M. Antonsen, Jr., and V.L. Granatstein. Overlapping and stochasticity of electron trajectories in cyclotron masers// Phys. Rev. Lett., 2004, V. 93, 055101 . 